\title{
A perturbation approach for the modelling of eddy current nondestructive testing problems with differential probes
}

\author{
Ruth V. Sabariego and Patrick Dular
}

\begin{abstract}
A perturbation technique applied to the finite element modelling of eddy-current nondestructive testing (ECNDT) problems is developed for taking into account differential probes. It concerns a $h$-conform formulation. The source term of the formulation is directly determined by the projection of the unperturbed field in a relatively small region around the defect. The voltage change due to the presence of the flaw is calculated by performing an integral over the defect and a layer of elements in the exterior domain that touch its boundary. The considered test case involves a shielded differential probe scanning the surface of a metal specimen for the detection of flaws.
\end{abstract}

Index Terms - Perturbation method, finite element method, nondestructive testing, eddy currents

\section{INTRODUCTION}

In eddy-current nondestructive testing problems (ECNDT), a fast and accurate calculation of the probe response is often required for identifying the flaws from measured data. When the excitation is time-harmonic, the observed quantity is usually the impedance variation due to the presence of the defect.

Several variants of the volume integral method (VIM) have been reported in literature [1], [2]. Herein, defects can be represented by a distribution of current dipoles in its volume. A boundary element method, with a VIM description for the defect, is proposed in [3]. As only the crack is discretised, the calculations associated to different probe positions are very fast. However, these techniques become extremely expensive in case of more complicated geometries (other than infinite stabs or tubes with homogeneous and linear material parameters).

The finite element method [4] allows to overcome this drawback, but may require a dense discretization in the vicinity of the defect (large 3D mesh). The impedance (or voltage) change due to the defect is calculated as the difference of the impedance values with and without flaw. Further, calculations for different probe positions are performed independently, which is time consuming.

A $\boldsymbol{b}$-conform based finite element scheme that directly calculates the distortion of the eddy-current due to a flaw was proposed in [5]. The counterpart $\boldsymbol{h}-\phi$ formulation is

Manuscript received May 2, 2006. This work has been supported by the Belgian Science Policy (IAP P5/34) and the European project VERDICT.

Ruth V. Sabariego and Patrick Dular are with the Dept. of Electrical Engineering and Computer Science, Institut Montefiore, University of Liège, Belgium (e-mail: r.sabariego@ulg.ac.be). P. Dular is a Research Associate with the Belgian National Fund for Scientific Research (F.N.R.S.). treated by the authors in [6]. Herein, the computation is split into a computation without flaw and a computation of the field distortion due to its presence. The unperturbed field is calculated in a large region, and applied as a source in the flaw for the second computation. The perturbed field can thus be determined in a reduced domain around the defect, what allows for an adapted discretization.

This paper deals with an extension of the method for taking into account differential probes. Further, an expression for the voltage variation is derived. As test case, we consider a piece with a crack and a shielded differential probe that scans its surface. The computational cost for both the conventional FE approach and the FE perturbation method will be discussed.

\section{Perturbation method}

The unperturbed magnetic field $\boldsymbol{h}_{u}$ is obtained by solving the $\boldsymbol{h}$-conform magnetodynamic formulation [7] in the whole domain. Then we consider a relatively small region around the defect without the probe. Note that the mesh of this reduced region is completely independent from the mesh of the whole domain and thus better adapted to the dimensions of the defect.

We consider a magnetodynamic problem in a bounded domain $\Omega$ (boundary $\Gamma$ ) of $\mathbb{R}^{3}$. The eddy current conducting part of $\Omega$ is denoted $\Omega_{c}$ and the non-conducting one $\Omega_{c}^{C}$ $\left(\Omega=\Omega_{c} \cup \Omega_{c}^{C}\right)$. Source conductors, with a given current density $\boldsymbol{j}_{s}$, are comprised in $\Omega_{s} \subset \Omega_{c}^{C}$. A flaw $\Omega_{f}$ (boundary $\Gamma_{f}$ ) appears in $\Omega_{c}$. The source of the perturbation problem in $\Omega_{f}$ is calculated through a projection method of the eddycurrent distribution without defect in the reduced domain.

For the sake of simplicity, let us assume hereafter a zeroconductivity flaw $\sigma_{f}=0$ with the same magnetic permeability $\mu_{f}=\mu$ as the host material $\Omega_{c}$. Further both are considered to be linear and isotropic. Let us particularise the Ampère law for the unflawed (subscript $u$ ) and flawed (subscript $f$ ) arrangements, i.e. $\operatorname{curl} \boldsymbol{h}_{u}=\sigma \boldsymbol{e}_{u}$ and $\operatorname{curl} \boldsymbol{h}_{f}=0$, where $\boldsymbol{h}$ and $e$ are the magnetic and electric fields. Subtracting these two expressions, the source is described as an electric current density in the flaw generating the perturbation, i.e.

$$
\boldsymbol{j}_{s f}=\operatorname{curl} \boldsymbol{h}=-\sigma \boldsymbol{e}_{u}=-\operatorname{curl} \boldsymbol{h}_{u}
$$

with $\boldsymbol{h}=\boldsymbol{h}_{f}-\boldsymbol{h}_{u}$ the perturbation magnetic field [6]. The extension of the formulation to other cases is straightforward. 


\section{A. $\boldsymbol{h}$-conform magnetodynamic formulation}

Adopting the magnetic field formulation, the general expression of the magnetic field $\boldsymbol{h}$ in $\Omega$ is $\boldsymbol{h}=\boldsymbol{h}_{s}+\boldsymbol{h}_{r}$, with $\boldsymbol{h}_{s}$ a source magnetic field in $\Omega$ satisfying $\operatorname{curl} \boldsymbol{h}_{s}=\boldsymbol{j}_{s}$ and $\boldsymbol{h}_{r}$ the reaction magnetic field in $\Omega_{c}$. In the non-conducting region $\Omega_{c}^{C}$, the reaction field $\boldsymbol{h}_{r}$ can be derived from a scalar potential $\phi$ such that $\boldsymbol{h}_{r}=-\operatorname{grad} \phi$.

The $\boldsymbol{h}-\phi$ magnetodynamic formulation is obtained from the weak form of the Faraday law:

$$
\begin{array}{r}
\partial_{t}\left(\mu \boldsymbol{h}, \boldsymbol{h}^{\prime}\right)_{\Omega}+\left(\sigma^{-1} \operatorname{curl} \boldsymbol{h}, \operatorname{curl} \boldsymbol{h}^{\prime}\right)_{\Omega_{c}}+\left\langle\boldsymbol{n} \times \boldsymbol{e}, \boldsymbol{h}^{\prime}\right\rangle_{\Gamma}=0, \\
\forall \boldsymbol{h}^{\prime} \in F_{h \phi}(\Omega)
\end{array}
$$

where $\boldsymbol{n}$ is the outward unit normal vector on $\Gamma$, part of the boundary of $\Omega ;(\cdot, \cdot)_{\Omega}$ and $\langle\cdot, \cdot\rangle_{\Gamma}$ denote a volume integral in $\Omega$ and a surface integral on $\Gamma$ of the product of their arguments; $F_{h \phi}(\Omega)$ is the function space defined on $\Omega$ and containing the basis functions for $\boldsymbol{h}$ (coupled to $\phi$ ) as well as for the test function $\boldsymbol{h}^{\prime}$ [7]. At the discrete level, this space is built with edge finite elements. The trace of $e$ is a constraint associated with $\Gamma$ (this constraint can e.g. be associated with a homogeneous natural boundary condition or with a global quantity) [7].

The unperturbed field $\boldsymbol{h}_{u}$ (with $\Omega_{f} \subset \Omega_{c}$ ) is obtained by particularising ( $\boldsymbol{h}=\boldsymbol{h}_{u}$ ) and solving (2). This field $\boldsymbol{h}_{u}$ is then projected on a reduced domain $\Omega^{\prime} \subset \Omega$ around the defect. Note that projecting only $\boldsymbol{h}_{u}$ is not sufficient as this way the local current $\boldsymbol{j}_{u}=\operatorname{curl} \boldsymbol{h}_{u}$ will not be conserved. Furthermore, the trace of source field $\boldsymbol{n} \times \boldsymbol{h}_{s f}$ on $\Gamma_{f}\left(\boldsymbol{h}_{s f}=-\boldsymbol{h}_{u}\right)$ contributes to the exterior domain $\Omega^{\prime} \backslash \Omega_{f}$. Indeed, the following interface condition has to be satisfied on $\Gamma_{f}$,

$$
\left.\boldsymbol{n} \cdot \boldsymbol{j}\right|_{\Gamma_{f}}=-\left.\boldsymbol{n} \cdot \boldsymbol{j}_{s f}\right|_{\Gamma_{f}},
$$

which is equivalent to considering

$$
\boldsymbol{n} \times\left.\boldsymbol{h}\right|_{\Gamma_{f}}=-\boldsymbol{n} \times\left.\operatorname{grad} \phi\right|_{\Gamma_{f}}+\boldsymbol{n} \times\left.\boldsymbol{h}_{s f}\right|_{\Gamma_{f}} .
$$

The source of the perturbation problem in $\Omega_{f}$ is calculated through a projection method in $\Omega^{\prime}$ as

$$
\begin{array}{r}
\left(\operatorname{curl} \boldsymbol{h}_{s f}, \operatorname{curl} \boldsymbol{h}^{\prime}\right)_{\Omega^{\prime}}-\left(\boldsymbol{j}_{s f}, \operatorname{curl} \boldsymbol{h}^{\prime}\right)_{\Omega^{\prime}}=0, \\
\forall \boldsymbol{h}^{\prime} \in F_{h \phi}\left(\Omega^{\prime}\right),
\end{array}
$$

where a gauge condition using a tree-cotree method at the discrete level in $\Omega^{\prime}$ is applied to ensure the uniqueness of the solution. The circulation of $\boldsymbol{h}_{s f}$ on the edges of $\Omega^{\prime} \backslash \Omega_{f}$ is fixed to zero. For the sake of conciseness, hereafter we refer to $\Omega^{\prime}$ as $\Omega$.

Taking into account the source in the flaw (1), the perturbation problem is completely characterised by (2) applied to the perturbation field $\boldsymbol{h}$ as follows:

$$
\begin{array}{r}
\partial_{t}\left(\mu \boldsymbol{h}, \boldsymbol{h}^{\prime}\right)_{\Omega}+\left(\sigma^{-1} \operatorname{curl} \boldsymbol{h}, \operatorname{curl} \boldsymbol{h}^{\prime}\right)_{\Omega_{c} \backslash \Omega_{f}}+\partial_{t}\left(\mu \boldsymbol{h}_{s f}, \boldsymbol{h}^{\prime}\right)_{\Omega} \\
+\left(\boldsymbol{j}_{s f}, \operatorname{curl} \boldsymbol{h}^{\prime}\right)_{\Omega_{f}}=0, \forall \boldsymbol{h}^{\prime} \in F_{h \phi}(\Omega) .
\end{array}
$$

\section{B. Calculation of the voltage variation}

The final goal is to calculate the voltage variation of the differential coil which allows us to detect and characterize the defect. However, the variation of the observed quantity is usually under $1 \%$ of the total value or even smaller in practical cases. The accurate calculation of this voltage variation $\Delta U$ is crucial.

Taking into account the developments presented in [6], a suitable definition (position of the cut of the coils linked to the direction of the current [7]) allow us to directly calculate the variation of the voltage $\Delta U$ between the unflawed and flawed problem. It can be accurately obtained by performing an integral over the defect $\Omega_{f}$ and a layer of elements in $\Omega \backslash \Omega_{f}$ that touch $\Gamma_{f}$.

A suitable treatment of the surface integral term in (2) consists in naturally defining a global voltage $U$ ( $\Delta U$ for differential probes) in a weak sense. We can define a global test function for $\boldsymbol{h}$ with a unit circulation along any current tube of the inductor so that the surface integral in (2) can be expressed as the product of a global voltage $U$ and a unit global current $I\left(\operatorname{curl} \boldsymbol{h}^{\prime}\right)$ [7].

Let us specify (2) for the unflawed problem, it holds

$$
\begin{aligned}
\partial_{t}\left(\mu \boldsymbol{h}_{u}, \boldsymbol{h}^{\prime}\right)_{\Omega}+\left(\sigma^{-1} \operatorname{curl} \boldsymbol{h}_{u}, \operatorname{curl} \boldsymbol{h}^{\prime}\right)_{\Omega_{c}} & =U_{u} I\left(\operatorname{curl} \boldsymbol{h}^{\prime}\right), \\
& \forall \boldsymbol{h}^{\prime} \in F_{h \phi}(\Omega) .
\end{aligned}
$$

Analogously, for the flawed problem, we can write

$$
\begin{aligned}
& \partial_{t}\left(\mu \boldsymbol{h}_{f}, \boldsymbol{h}^{\prime}\right)_{\Omega}+\left(\sigma^{-1} \operatorname{curl} \boldsymbol{h}_{f}, \operatorname{curl} \boldsymbol{h}^{\prime}\right)_{\Omega_{c} \backslash \Omega_{f}}+ \\
& \left(\boldsymbol{e}_{f}, \operatorname{curl} \boldsymbol{h}^{\prime}\right)_{\Omega_{f}}=U_{f} I\left(\operatorname{curl} \boldsymbol{h}^{\prime}\right), \quad \forall \boldsymbol{h}^{\prime} \in F_{h \phi}(\Omega),
\end{aligned}
$$

where we have added the term $\left(\boldsymbol{e}_{f}, \operatorname{curl} \boldsymbol{h}^{\prime}\right)_{\Omega_{f}}$ which is not cancelled as in the general case due to the imposed perturbation current in the flaw, i.e. $\operatorname{curl} \boldsymbol{h}^{\prime} \neq 0$ in $\Omega_{f} \subset \Omega_{c}^{C}$.

Choosing as test functions $\boldsymbol{h}^{\prime}=\boldsymbol{h}_{f}$ in (7) and $\boldsymbol{h}^{\prime}=\boldsymbol{h}_{u}$ in (8) and subtracting (7) from (8), we obtain

$$
\begin{aligned}
\Delta U I & =\left(U_{f}-U_{u}\right) I= \\
& =-\left(\sigma^{-1} \operatorname{curl} \boldsymbol{h}_{u}, \operatorname{curl} \boldsymbol{h}_{f}\right)_{\Omega_{f}}+\left(\boldsymbol{e}_{f}, \operatorname{curl} \boldsymbol{h}_{u}\right)_{\Omega_{f}}= \\
& =\left(\boldsymbol{e}_{f}, \operatorname{curl} \boldsymbol{h}_{u}\right)_{\Omega_{f}},
\end{aligned}
$$

where the first volume integral cancels because $\boldsymbol{h}_{f}$ is curl-free in $\Omega_{f}$ and $I$ is the real current injected in the inductor.

The perturbed electric field $e_{f}$ is not known in the flaw but can be calculated by means of (8) with $\boldsymbol{h}^{\prime}=\boldsymbol{h}_{s f}$. This way $I\left(\operatorname{curl} \boldsymbol{h}^{\prime}\right)=0$ and the voltage variation $\Delta U$ is obtained as

$$
\begin{aligned}
\Delta U I & =\left(\boldsymbol{e}_{f}, \operatorname{curl} \boldsymbol{h}_{u}\right)_{\Omega_{f}}=-\left(\boldsymbol{e}_{f}, \operatorname{curl} \boldsymbol{h}_{s f}\right)_{\Omega_{f}}= \\
& =\left(\sigma^{-1} \operatorname{curl}\left(\boldsymbol{h}+\boldsymbol{h}_{s f}\right), \boldsymbol{j}_{s f}\right)_{\Omega_{c} \backslash \Omega_{f}}+\partial_{t}\left(\mu\left(\boldsymbol{h}+\boldsymbol{h}_{s f}\right), \boldsymbol{h}_{s f}\right)_{\Omega},
\end{aligned}
$$

where the domain of integration, at the discrete level, is actually limited to $\Omega_{f}$ and a layer of elements in $\Omega \backslash \Omega_{f}$ touching $\Gamma_{f}$ due to the definition of $\boldsymbol{h}_{s f}$.

Consequently, no integration of any flux variation in the coils is required, which would not be directly accessible because of the lack of explicit solution there (no mesh of the coils for the perturbed problem). 


\section{Application EXAmple}

We consider a Titanium piece $(\sigma=1.798 \mathrm{MS} / \mathrm{m}$, Fig. 1) with a semi-cylindrical crack ( $1 \mathrm{~mm}$ long, $0.2 \mathrm{~mm}$ wide and $0.1 \mathrm{~mm}$ deep) at the upper border. A magnetically shielded differential probe scans the outer surface (Fig. 2). It consists of a driver coil carrying an imposed sinusoidal current of $1 \mathrm{~A}$ and frequency $f=200 \mathrm{kHz}$, that surrounds two D-shaped sensing coils wound around a ferrite core $\left(\mu_{r}=1000\right)$. The liftoff between the coil and the surface of the piece is $0.1 \mathrm{~mm}$.

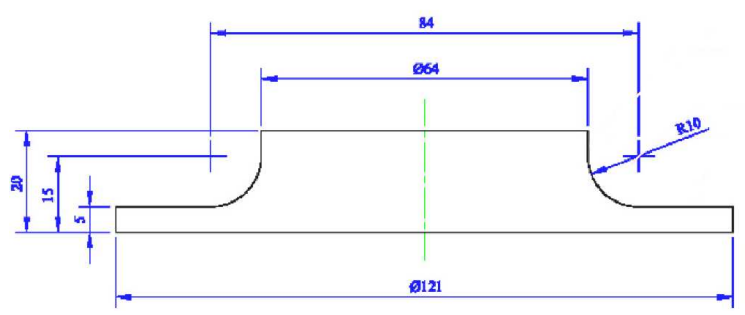

Fig. 1. Cut of the Titanium piece with defect at the upper border
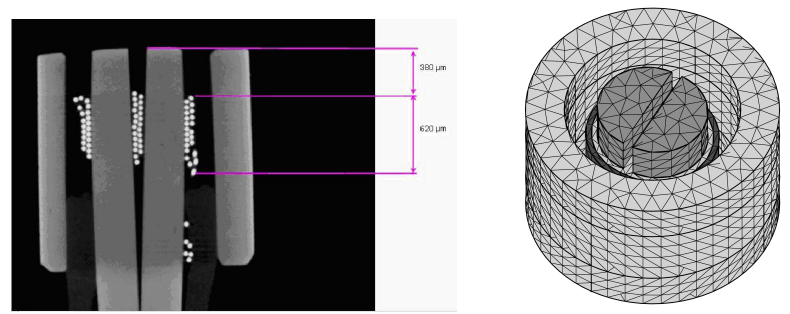

Fig. 2. Longitudinal cut (left) and mesh (right) of the shielded differential probe: driver coil surrounding two D-shaped sensing coils

We are interested in calculating the difference in voltage $\Delta U$ of the two sensing coils that comprises the differential probe. A single source field (used as the test function $\boldsymbol{h}^{\prime}$ in (2)) associated to these coils connected in series allows us to directly calculate $\Delta U$ in both the unflawed $\Delta U_{u}$ and flawed problem $\Delta U_{f}$ [7].
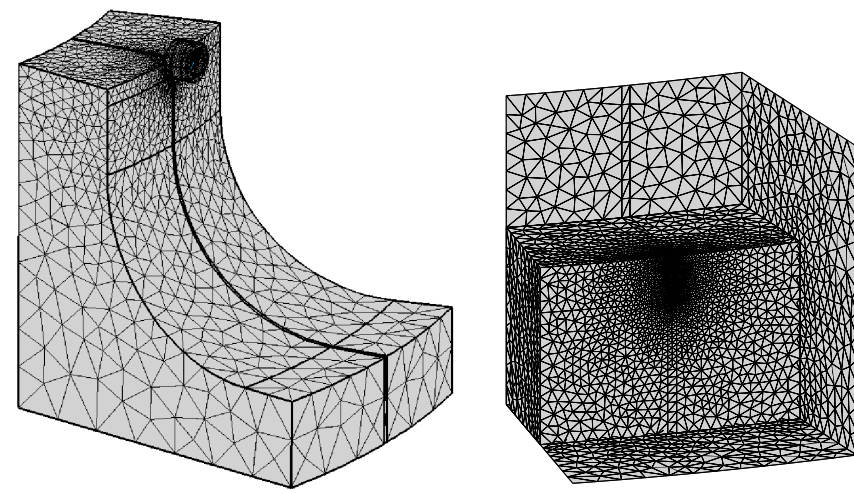

Fig. 3. 3D FE mesh of both the complete domain $\Omega$ (left) and the reduced domain $\Omega^{\prime}$ of size $D=3.5 \delta$ used in the perturbation method. The source field is defined in the flaw, the excitation coil is taken as air.
We define the size of the reduced domain $\Omega^{\prime}$ in terms of the distance $D$ (multiple of the skin depth $\delta=1 / \sqrt{\pi f \mu \sigma}=$ $0.84 \mathrm{~mm}$ of the tube) from the boundary of the crack to the boundary of $\Omega^{\prime}$. The size of the reduced domain $\Omega^{\prime}$ is chosen taken into account the $2 \mathrm{D}$ results presented in [6] and depicted for convenience in Figs. 4 and 5. Herein, we vary the dimensions of $\Omega^{\prime}$ around the defect in terms of $D$ and compare the voltage variation $\Delta U$ obtained with both the conventional approach (the unflawed and the flawed problems are solved successively) and the proposed perturbation method (directly integrating in a sub-domain of $\Omega^{\prime}$ ). A correlation between the parameter $D$ and the accuracy of the results can then be established.

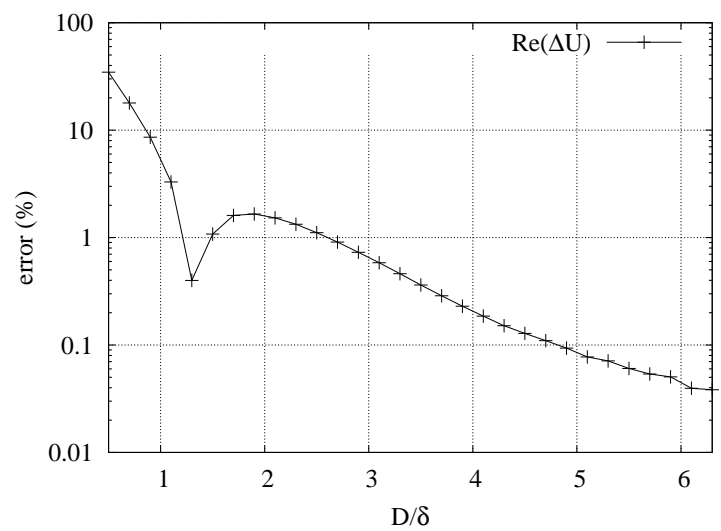

Fig. 4. Relative error (\%) in the real part of $\Delta U$ as a function of the normalised size of $\Omega^{\prime}$

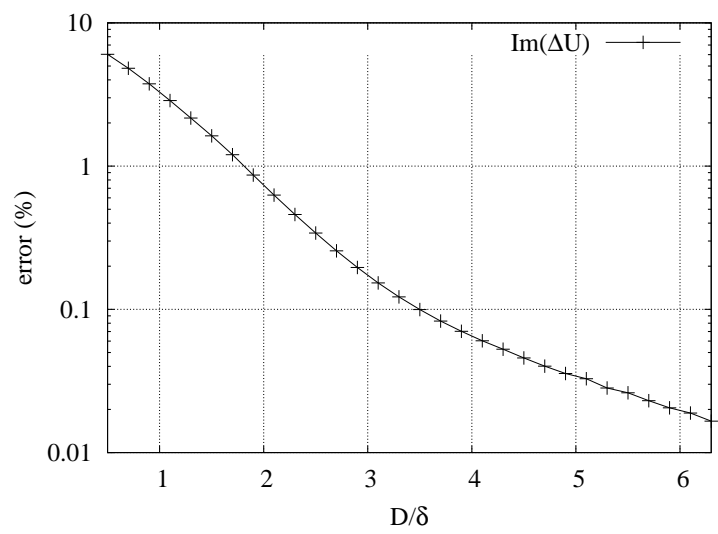

Fig. 5. Relative error (\%) in the imaginary part of $\Delta U$ as a function of the normalised size of $\Omega^{\prime}$

One observes in Figs. 4 and 5 that the error in the real and imaginary parts of $\Delta U$ is smaller than $1 \%$ for $D \geq 3 \delta$ and $D \geq 2 \delta$, respectively. Therefore, we take a reduced domain $\Omega^{\prime}$ of size $D=3.5 \delta$ for our 3D computations.

The FE discretisation of the complete domain $\Omega$, with 107186 tetrahedra, yields 85676 unknowns for the unperturbed problem and 83008 unknowns for the perturbed problem. The FE discretisation of the reduced domain $\Omega^{\prime}$, with 50085 tetrahedra, yields 73072 unknowns for the perturbation problem. 
Note that the discretisation of the reduced domain $\Omega^{\prime}$ is finer and better adapted to the dimensions of the crack (Fig. 3).

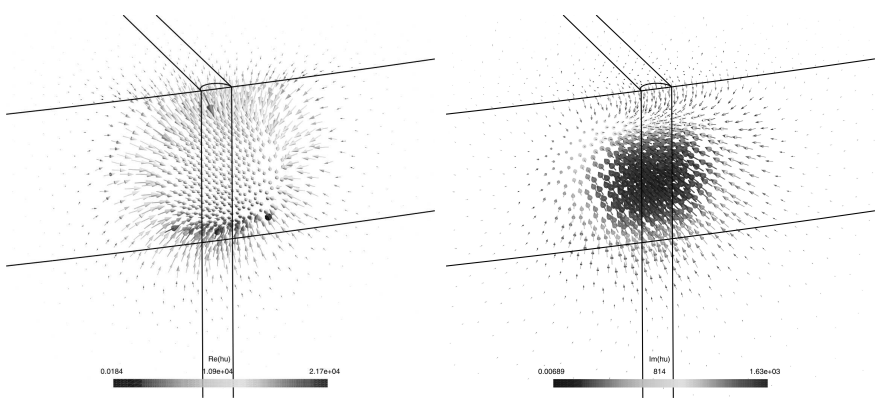

Fig. 6. Real and imaginary part of unperturbed magnetic field

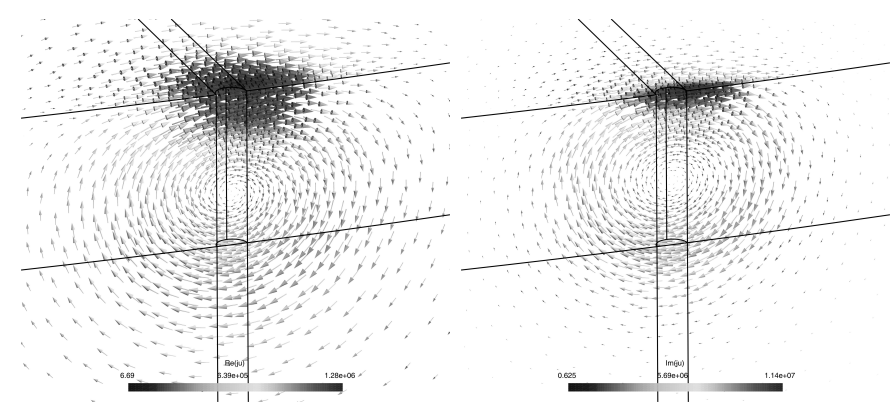

Fig. 7. Real and imaginary part of unperturbed current density

The real and imaginary parts of the unperturbed magnetic field, with the coil centered at the center of the flaw, are depicted in Fig. 6. The real and imaginary parts of the unperturbed electric current density are shown in Fig. 7. The real and imaginary parts of the perturbation source current imposed in the flaw $\Omega_{f}$ in the perturbation method are represented in Fig. 8 .
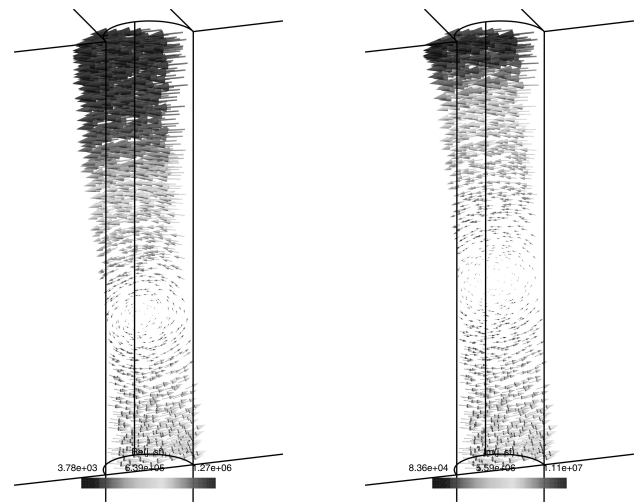

Fig. 8. Real and imaginary part of the perturbation source current density imposed in the flaw $\Omega_{f}$

The voltage variation obtained with the traditional approach is $\Delta U=-0.402+\imath 0.113 \mathrm{mV}$. When applying the proposed perturbation method, we achieve $\Delta U=-0.406+\imath 0.114 \mathrm{mV}$. The error in both the real and imaginary parts is thus approximately $1 \%$.

\section{A. Computational cost}

The system of algebraic equations is solved by means of the iterative solver GMRES [8] with ILU-preconditioning on a $2.26 \mathrm{GHz}$ Intel Pentium M Processor.

The FE conventional approach requires performing two successive computations on the same mesh of the complete domain described above. The unperturbed and perturbed problem are solved after $4605 \mathrm{~s}$ and $4256 \mathrm{~s}$, respectively. The total computation time equals thus $8861 \mathrm{~s}$.

When applying the perturbation scheme, the two given meshes are needed: the mesh of the complete domain $\Omega$ and the finer mesh of the reduced domain $\Omega^{\prime}$. The solution of the perturbation method is obtained after $6241 \mathrm{~s}$. Therefore, the savings in memory reach approximately $30 \%$. This reduction illustrates the efficiency of the method.

\section{CONCLUSION}

A 3D FE perturbation technique based on the $\boldsymbol{h}$-conform magnetodynamic formulation has been elaborated. The unperturbed field is conventionally calculated in the complete domain taking advantage of any symmetry or analytical solution and applied as a source in the flaw. The perturbed field is then determined in a reduced domain surrounding the defect. Its discretisation is thus chosen independently of the dimensions of the probe and the specimen under study and thus better adapted.

Furthermore, the voltage variation due to the presence of the flaw is efficiently obtained by performing an integral over the defect and a layer of elements in the exterior domain that touch its boundary. Therefore no integration of any flux variation in the coils is required. Significant savings in computation time are achieved.

\section{REFERENCES}

[1] J. R. Bowler and S. A. Jenkings, "Eddy-current probe impedance due to a volumetric flaw," Journal of Applied Physics, vol. 70, no. 3, pp. 1107-1114, 1991.

[2] D. Prémel, G. Pichenot, and T. Sollier, "Development of a 3D electromagnetic model for eddy current tubing inspection: application to the simulation of probe eccentricity," Int. J. Appl. Electromag. Mech., vol. 19, no. $1-4$, pp. 521-525, 2004

[3] O. Michelsson and F. H. Uhlmann, "A boundary-integral based forward solution for eddy current nondestructive testing," IEEE Transactions on Magnetics, vol. 36, no. 4, pp. 756-759, 2000.

[4] N. Ida and W. Lord, "A finite element model for three-dimensional eddy current NDT phenomena," IEEE Transactions on Magnetics, vol. 21, no. 6, pp. 2635-2643, 1985.

[5] Z. Badics, Y. Matsumoto, K. Aoki, F. Nakayasu, M. Uesaka, and K. Miya, "An effective 3-D finite element scheme for computing electromagnetic field distorsion due to defects in eddy-current nondestructive evaluation," IEEE Transactions on Magnetics, vol. 33, no. 2, pp. 1012-1020, 1997.

[6] R. V. Sabariego and P. Dular, "A perturbation technique for the finite element modelling of nondestructive eddy current testing," in Electromagnetic Fields in Mechatronics, Electrical and Electronic Engineering, ser. Studies in Applied Electromagnetics and Mechanics, Vol. 27. A Krawczyk, S. Wiak and X. M. Lopez-Fernandez (Eds.), IOS Press (Amsterdam), 2006.

[7] P. Dular, P. Kuo-Peng, C. Geuzaine, N. Sadowski, and J. P. A. Bastos, "Dual magnetodynamic formulations and their source fields associated with massive and stranded inductors," IEEE Transactions on Magnetics, vol. 36, no. 4, pp. 1293-1299, 2000.

[8] Y. Saad and M. H. Schultz, "GMRES: A Generalized Minimal Residual Algorithm for solving nonsymmetric linear systems," SIAM J. Sci. Comput., vol. 7, no. 3, pp. 856-869, July 1986. 